International Journal of Child, Youth and Family Studies (2016) 7(3/4): 324-342

DOI: http://dx.doi.org/10.18357/ijcyfs73-4201616088

\title{
A LITERATURE REVIEW OF STRATEGIES FOR THE PREVENTION OF INTIMATE PARTNER VIOLENCE DURING THE CHILDBEARING YEARS
}

\section{Teila Sinnott and Sibylle Artz}

\begin{abstract}
This narrative literature review examines the peer-reviewed research in English on the prevention of intimate partner violence (IPV) during the childbearing years, published between January 2000 and January 2015. It shows that in the 43 articles that were located there is a disproportionate emphasis on making females responsible for not becoming victims of IPV, especially if they are mothers, who are made responsible for ensuring not only their own but also their children's safety. Also noted is a striking lack of research on the prevention of IPV perpetration in males, particularly fathers. Even the current IPV research that focuses on interventions with males, notes the absence of interventions specifically designed for fathers and calls for both a better understanding of the underlying factors that contribute to male violence and a focus on interventions tailored to preparing men for the challenges of fatherhood. Two such programs are described in the final section of this review.
\end{abstract}

Keywords: intimate partner, domestic violence, domestic abuse, prevention, childbearing, pregnancy, parenthood, transition to parenthood, father, fatherhood, paternal

Teila Sinnott, MA is working for the Government of Alberta as a policy analyst as part of a policy internship program. Email: teilasinnott@gmail.com

Sibylle Artz, PhD (the corresponding author) is a Professor in the School of Child and Youth Care at the University of Victoria, P.O. Box 1700, STN CSC, Victoria, British Columbia, Canada, V8W 2Y2. Email: sartz@uvic.ca 
Interest in the serious and widespread issue of intimate partner violence (IPV) has increasingly been characterized by a growing concern for the impact of exposure to this violence on children (Jackson \& Artz, 2014) Although children may not be the direct victims of IPV, research clearly shows that witnessing violence between family members puts children at risk of adverse outcomes in multiple domains, including neurological, mental, and physiological development; these in turn can lead to poor physical health; multiple behavioural challenges such as conduct disorder, delinquency, and crime, and, eventually, poor academic and employment outcomes ( Artz et al., 2014). The personal impacts of witnessing IPV also come with enormous economic costs to society: costs for the provision of medical care, criminal justice, and other social services, and costs incurred through the loss of productivity in education and the workplace (Andresen \& Linning, 2014). Estimates that put the rate of IPV exposure at 125,000 new children in Canada each year approximate a yearly economic expense of $\$ 759$ million for a single cohort of children (Andresen \& Linning, 2014). New costs of course are not “freestanding” and are added each year to the already accumulated lifelong costs of all previous new cases. With \$759 million as the benchmark for each new cohort and the cumulative continuing costs over a ten-year period for the ten cohorts involved, the total costs of an additional cohort year over year is $\$ 26.4$ billion (Andresen \& Linning, 2014). Taken together, the serious and far-reaching consequences of childhood exposure to IPV suggest to us that more should be done to prevent IPV and children's exposure to this toxic experience. With that in mind we undertook a review of the current literature on research into the prevention of IPV in families with children.

While we acknowledge that violent behaviours can be perpetrated by either member of an opposite-sex couple, our review focuses primarily on male violence against women because females are much more likely to be the victims of family, spousal, and IPV than are males (Canadian Centre for Justice Statistics, 2015; Sinha, 2012, 2013). Of particular note is that the literature we examined places a disproportionate emphasis on making females responsible for not becoming victims of IPV. This was especially true for mothers, who were considered responsible for ensuring not only their own but also their children's safety. We also found a striking lack of research on the prevention of IPV perpetration in males, particularly fathers. Thus, while maintaining our focus on identifying effective IPV prevention strategies, we also assessed the current landscape of IPV prevention practices, and the effects of these current practices in positioning mothers as responsible for managing IPV-related behaviour and outcomes.

\section{Methodology}

To locate the existing body of research for this review, a computerized literature search was conducted. Our central search terms - intimate partner violence, domestic violence, and domestic abuse - were variously combined with the keywords prevention, childbearing, pregnancy, parenthood, and transition to parenthood, and entered into Academic Search Complete, Google Scholar, PSYCInfo, Social Sciences Full Text, and Web of Science. Titles and abstracts identified in these initial searches were reviewed for relevance. The reference lists of these eligible 
International Journal of Child, Youth and Family Studies (2016) 7(3/4): 324-342

articles were also reviewed to find additional relevant articles that may not have been identified through the electronic search.

Our inclusion criteria were as follows: (a) focus on childbearing years or transition to parenthood (prenatal to first five years); (b) discussion of strategies for the prevention of IPV, or intervention in existing IPV such that intervention would preclude the risk of exposure for children; (c) published in the English language; (d) published between January 2000 and January 2015; and (e) published in a peer-reviewed journal. Systematic reviews and study protocols were eliminated; however, relevant non-intervention studies and non-research commentaries were retained, as they helped to build a more comprehensive understanding of the current state of IPV prevention during childbearing years. Following this initial search, a total of 31 articles met the inclusion criteria. Of this number, 29 articles studied screening techniques or interventions for IPV victims in medical care settings or through home visitation programs, and 2 articles evaluated interventions for couples during the transition to parenthood. This initial search did not yield any literature on IPV prevention specifically for men during the transition to fatherhood.

Our finding that there appears to be a significant lack of research on preventing perpetration of IPV by men who are about to become fathers, and a concomitant emphasis on helping women to avoid violent men, led us to expand our search terms and inclusion criteria. Using the same databases, our second search attempt sought to apply new search terms that focused more specifically on IPV in the context of fatherhood (e.g., father, fatherhood, paternal). In order to draw in some research and commentary that would help us learn more about the configuration of contemporary IPV prevention practices, we included articles that focused on IPV intervention specifically within the context of fatherhood, even following children's exposure to violence. Using this adjusted search strategy, we were successful in identifying an additional 12 articles. Although these modifications departed somewhat from our original intention to examine the prevention of IPV before children's exposure, the additional articles that were identified allowed for a more rounded understanding of the current landscape of IPV research specifically as it pertains to homes with children, which was consistent with our primary aim. Notably, even with the expanded inclusion criteria for IPV perpetrator research, our searches returned a considerably smaller body of research than that on preventing women's victimization by IPV.

\section{Main Areas of Intervention}

\section{Screening during Pregnancy}

The majority of the articles that we located in our search focused on screening and intervention for IPV in the context of medical practice, and this was especially true with regard to pregnant women. We believe that this may reflect a response to research showing that violence during pregnancy may be more severe and more frequent, like the work of Bianchi and colleagues (2014), who found that women reporting abuse during pregnancy had significantly higher scores for threat of abuse, physical abuse, and danger for murder than women who reported IPV outside of pregnancy. It is also possible that research tends to focus on pregnant women because 
International Journal of Child, Youth and Family Studies (2016) 7(3/4): 324-342

experiencing IPV during gestation can endanger the health of both the women and their unborn children (Bianchi et al., 2014). Additionally, maternal medical care typically involves women in ongoing interactions with health care providers, thus providing a key opportunity for intervention (Bacchus, Mezey, Bewley, \& Haworth, 2004).

Several studies that we reviewed aimed to determine the demographic and other factors that were associated with women who reported IPV while pregnant, in order to create a pregnancyfocused IPV risk profile that could be used to help maternal care practitioners identify cases early and provide timely assistance to victims. Some common findings among these studies were that women who reported experiencing IPV during pregnancy were typically young, unmarried, nonwhite, lacking social supports, and afraid of someone close to them (Andersen, Marshak, \& Hebbeler, 2002; Rådestad, Rubertsson, Ebeling, \& Hildingson, 2004; Reichenheim, Patricio, \& Moraes, 2008). Also, medically-focused symptoms and presenting complaints such as back pain, chronic illness, coital pain, depression-related symptoms, stomach pain, and urinary tract problems were noted as specific indicators of possible IPV during pregnancy (Rådestad et al., 2004). Other IPV-related difficulties identified in the research included experiencing depression or anxiety, child sexual abuse (Andersen et al., 2002), drug use, and a history of abortion (Reichenheim et al., 2008). The authors of these studies suggest that asking about these symptoms and recognizing them as risk factors can support clinicians in identifying the emergence of IPV during pregnancy so that appropriate steps can be taken to intervene and prevent further abuse and harm.

A study conducted in the United Kingdom that assessed the prevalence of IPV in pregnancy found that $2.5 \%$ of pregnant women reported IPV if asked about it directly during a routine interview with a midwife (Bacchus et al., 2004). The evidence from this study suggests that the introduction of routine screening of pregnant women could significantly contribute to increased rates of IPV detection, as women rarely disclose IPV to health care professionals unless directly asked. In establishing the most effective IPV screening methods with pregnant women, a Japanese study determined that self-administered questionnaires, as opposed to face-to-face interviews, resulted in significantly higher identification rates (Kataoka, Yaju, Eto, \& Horiuchi, 2010). This is a useful finding; however, further studies are needed in different regional and cultural settings to establish the generalizability of the results, particularly as Japanese cultural norms regarding verbal disclosure of private and family matters may have influenced these outcomes (Kataoka et al., 2010).

Given the importance of directly asking potential victims about IPV, Humphreys, Tsoh, Kohn, and Gerbert (2011) investigated whether an interactive video-counselling intervention presented during prenatal care, with a cue sheet for practitioners to enquire about IPV, would improve rates of IPV identification. Women receiving the video counselling were significantly more likely to report having a discussion with their care provider about IPV than the women receiving the usual care (Humphreys et al., 2011). Although this study only reported on providerpatient discussions of IPV and did not indicate rates of successful identification of IPV, this 
International Journal of Child, Youth and Family Studies (2016) 7(3/4): 324-342

strategy may nonetheless provide a means of overcoming some of the documented barriers to greater prevalence of screening and risk assessment (Humphreys et al., 2011).

Practitioners' attitudes towards and knowledge about IPV have also been identified as playing a crucial role in IPV screening and response. A comparison of hospital- and communitybased midwives' views and experiences with routine IPV enquiry indicated that both groups of midwives felt that they had a significant role to play in responding to IPV (Lazenbatt, Taylor, \& Cree, 2009). However, both groups also tended to underestimate the prevalence of IPV and only identified and responded to a portion of these cases (Lazenbatt et al., 2009). This suggests a need for more training for maternal care providers to gain knowledge and confidence in responding to IPV. The Bristol Pregnancy Domestic Violence training program for midwives is one example of a program that has been developed in response to such a need (Salmon, Murphy, Baird, \& Price, 2006; Baird, Salmon, \& White, 2013). An evaluation of the Bristol program found it to be successful in improving midwives’ knowledge, skills, and attitudes regarding routine antenatal enquiry for IPV (Salmon et al., 2013), and in a five-year follow-up, showed a statistically significant increase in midwives' self-reported confidence and knowledge about responding to disclosure compared to the original data (Baird et al., 2013). Despite increases in knowledge and confidence, however, the actual rates of inquiry following delivery of the program were not as high as anticipated (Baird et al., 2013). Midwives identified several significant barriers that have limited greater implementation of routine screening, suggesting that changes in practitioner knowledge and attitudes are only one part of the equation.

The most widely documented barrier to greater screening was the presence of a partner during antenatal consultation, which prevented maternal care staff from being able to ask directly about IPV (Baird et al., 2013; Lazenbatt et al., 2006; Marchant, Davidson, Garcia, \& Parsons, 2001; Salmon et al., 2006). In addition, an analysis of policies and practices in midwife trusts in England and Wales found that only $12 \%$ of the units studied had written policies for identifying IPV, and only 30\% had any form of agreed practice in this area (Marchant et al., 2001). Similar findings have suggested that midwives have been reluctant to enquire about IPV because they lacked the requisite knowledge of organizational policies (Lazenbatt et al., 2006), or because of a perceived lack of organizational support, time, and resources (Salmon et al., 2006). In responding to known barriers, an earlier study by Marchant and colleagues (2001) recommended that, in addition to greater training and education, clear policies and guidelines focusing on the identification of IPV should be put in place in maternal care settings. Baird et al. (2013) also emphasized these recommendations and noted that, in particular, such policies should ensure that a woman is seen alone at least once during her pregnancy to give her an opportunity for safe disclosure.

\section{Intervention during Pregnancy}

Screening for IPV risk during pregnancy is becoming increasingly recommended as a best practice for the reduction and prevention of IPV victimization, but based on our review little is yet 
International Journal of Child, Youth and Family Studies (2016) 7(3/4): 324-342

known about which interventions are most effective in assisting women once IPV victimization has been recognized. Five studies were identified that discussed different treatment modalities employed with pregnant women. We present these below in the order in which they were published.

In seeking to compare the treatment outcomes for pregnant, physically abused Hispanic American women who were receiving assistance at two urban prenatal clinics, McFarlane, Soeken, and Wiist (2000) engaged 329 participants in a longitudinal, repeated-evaluation interview study that compared three types of intervention - brief therapy, counselling, and outreach - by interviewing participants at 2, 6, 12, and 18 months post-delivery. Analyzing their data using repeated measure ANOVA, they found that violence scores at 2 months were significantly lower for the outreach group $(\mathrm{p}<.05)$ and the brief therapy group than for the counselling group. However, they also found that this initial significantly lower finding for the outreach group disappeared over time so that no treatment differences remained at 6,12, and 18 months. Still, the 6-, 12-, and 18-month follow-ups showed that, despite the somewhat patchy initial results, positive findings did emerge over time, in that the severity of abuse had declined significantly for all three groups ( $p$.001), significantly bettering the initial immediate positive result for the outreach group. These long-term results can be seen as promising for all three interventions, but should be interpreted with caution since no control groups were involved.

In a Hong Kong-based, 110 participant, randomized control group study focused on empowerment training of pregnant women who were being abused by their intimate partners, Tiwari et al. (2005) found that treatment group participants who had been subjected to minor physical abuse reported significantly less psychological abuse and significantly fewer incidents of minor physical violence after receiving the intervention. However, women experiencing sexual or serious physical abuse reported no significant changes.

The empowerment approach also yielded some significant results in a subsequent study conducted in Peru by Cripe et al. (2010). The study, which involved 220 women randomly assigned to either empowerment treatment or a control group receiving standard care, consisted of asking about abuse and offering program referrals. Cripe and colleagues (2010) found that, compared to the control group, the empowerment treatment group "were more likely to hide money (44.6\% vs. 34.3\%), establish a code with family or friends (19.6\% vs. $16.2 \%)$, ask neighbors to call police if violence began (6.9\% vs. $1.0 \%)$, had available bank account numbers (17.1\% vs. 3.1\%), had valuable jewelry (8.4\% vs. 3.8\%), and had available a hidden bag with extra clothing (9.0\% vs. 3.1\%)” (p. 2054). The fact that the members of the intervention and control groups who participated in the study did not report significant differences in their increased adoption of safety behaviours, in their improved health-related quality of life, or in their increased use of community resources, suggests that care in general outweighs the importance of any particular therapeutic approach. As Cripe et al. state, "Simply asking pregnant women about abuse and offering referral could potentially interrupt and prevent further IPV” (p. 2072). 
Promising findings were also reported by Kiely, El-Mohandes, El-Khorazaty, and Gantz, (2010) in their 1044 participant, randomized control group study of pregnant or recently delivered African American women, where the intervention group received individually tailored psychobehavioural counselling sessions and the control group received standard prenatal care. Keily et al. found that overall, the women who had been randomly assigned to the counselling group reported significantly fewer episodes of IPV victimization than those who remained within the standard prenatal care group, although levels of severity of IPV yielded differences within the intervention group: those in the intervention group who had earlier experienced minor IPV had significant reductions in further IPV episodes both during pregnancy and postpartum, while women who had previously experienced severe IPV reported significant reductions in further episodes only during the postpartum period. As well, those who received the counselling intervention had fewer preterm infants and longer gestation periods, leading the researchers to conclude that, "A relatively brief intervention during pregnancy had discernible effects on IPV and pregnancy outcomes” (p. 273).

Acknowledging the problem and offering help and support are of central importance to violence prevention, a finding that is further supported by Kramer, Nobusch, and Rice (2012) who, in their evaluation of Safe Mom, Safe Baby, a collaborative, community-based initiative serving pregnant and newly delivered mothers who disclose IPV victimization during screening, found that the clients participating in the program "achieved birth outcomes comparable to the overall population of women delivering at the centre" (p. 314). While this study has limitations in that it did not involve a control group and did not provide data about the occurrence of IPV victimization, it is still noteworthy that women receiving the intervention reported a higher number of safety behaviours at program completion than they had reported at intake, and more than half indicated progress toward the maintenance of violence-free relationships.

Finally, we think it worthy of note that several studies that we have discussed in this section have suggested that the presence of an IPV assessment may itself contribute to reducing or halting future abuse by simply making women aware of this concern (Cripe et al., 2010; McFarlane et al., 2000; Tiwari et al., 2005); therefore, future research should seek to distinguish between outcomes prompted by screening alone and those that can be credited to specific intervention programs that could be generalized to multiple groups and locations.

\section{Pediatric Screening}

Because of the multiple negative outcomes associated with children's exposure to IPV, this issue has also come to the attention of pediatricians. As Thackeray, Hibbard, and Dowd (2010) note, "Pediatricians need to be aware that most abused caregivers will seek care for their children but not for themselves, which makes the pediatric setting an ideal place to be alert to the presence of IPV” (p.1096). The literature on pediatric care that we reviewed showed that, in pediatric just as in maternal care settings, there are several identified barriers to IPV screening; these include lack of time and resources, lack of knowledge about IPV, insufficient referral sources, and fear of 
offending or angering the caregiver (Erickson, Hill, \& Siegel, 2001; Thackeray et al., 2010). Studies that examined routine IPV screening report a range of findings, from $5 \%$ to $18 \%$ of participants reporting IPV in one study (Borowsky \& Ireland, 2002), and 8.5\% in another (Erickson et al., 2001). Further, pediatricians were found to significantly underestimate the prevalence of IPV in their practice (Erickson et al., 2001). Pediatricians did not uniformly inquire about IPV, and those most likely to conduct routine screenings were those who had previously completed IPV-specific training (Erickson et al., 2001). In response to this ongoing need, education about the dynamics of IPV and the implementation of an office protocol for managing cases of IPV - particularly one developed with input from local community resources and women's shelters — have been recommended (Borowsky \& Ireland, 2002; Erickson et al., 2001; Thackeray et al., 2010). There are, however, no identified studies that examine the effectiveness of pediatric screening on reducing IPV. This suggests that more research into pediatric screening is needed.

\section{Early Prevention Home Visiting Programs}

Early prevention home visiting (EPHV) programs that are part of pre- and post-natal care provide another opportunity for screening and intervention for potential victims of IPV. These programs have traditionally been used to identify home and caregiving risk factors in order to promote healthy family environments and facilitate positive child development (Chamberlain, 2008). In response to growing evidence about the negative impact of IPV on children, there has been an increased interest in adapting existing EPHV programs to specifically address risk of IPV and exposure of children (Chamberlain, 2008). In one randomized control study of home visitation following child birth, Hawaiian mothers receiving EPHV services reported lower rates of IPV victimization (e.g., being physical assaulted) and significantly lower rates of perpetration (e.g., physically assaulting their partners) than the control group of matched families who did not receive EPHV services (Bair-Merritt et al., 2010). A Dutch study of the impact on IPV of nurses' home visits during pregnancy reported findings similar to those of the Hawaiian study: at 32 weeks of pregnancy, women receiving the EPHV intervention reported significantly less psychological, physical, and sexual aggression by intimate partners, and significantly lower use of psychological and physical aggression towards and injury of their intimate partners, than the matched participants in the control group (Mejdoubi et al., 2013). Further, in the Dutch study, these significant reductions in both victimization and perpetration were sustained at 24 months after birth. Together, the two studies suggest that EPHV programs can assist with mitigating women's victimization and perpetration of IPV following childbirth.

Another aspect of EPHV programs that has been assessed is the impact of the type of visitor used. In a comparison of the impact of home visits performed by nurses and paraprofessionals, Olds et al. (2004) found in a two-year follow-up that only the nurse-visited intervention group reported a significant decrease in physical partner violence. Olds and colleagues suggest that this indicates that nurses may be more effective than paraprofessionals in dealing with IPV during home visits, although the reason for this remains unknown. As well, it is 
not yet possible to state definitively that one type of home visitor is more effective overall than another, since studies to date have involved a range of home visitors with a variety of educational training backgrounds and occupational titles. For example, Taft et al. (2011) examined the role of peer mentors, rather than professional home visitors, in providing social support to pregnant and recent mothers at risk of IPV victimization and found that at follow-up mean abuse scores favoured the intervention group; outcome measures for depression, well-being, and social support also improved, although only at weak levels. Still, these findings suggest that mentorship programs provide a promising approach for social support to new mothers. As the existing research on EPHV impact shows, drawing conclusions about the relationship between professional designation and impact is as yet somewhat premature and in need of further research.

Different types of home visitor, filling various roles, may have different confidence levels in dealing with IPV, which will have an impact on the effectiveness of the EPHV. Research has shown that visitors reported multiple personal barriers to addressing IPV during their visits. These included: (a) fear of offending the client and having the client withdraw from services; and (b) not knowing what to do if IPV were disclosed (Eddy, Kilburn, Chang, Bullock, \& Sharps, 2008; Jack et al., 2012; Sharps et al., 2013); (c) believing the formal training they received to be inadequate (Tandon, Parillo, Jenkins, \& Duggan, 2005); and (d) not knowing how to assist clients in connecting with the resources they needed due to a lack of knowledge about the community services available (Eddy et al., 2008). As reported by Jack et al. (2012), home visitors' lack of general knowledge and skills in identifying and responding to IPV was certainly noted by their clients. For these reasons, Jack and colleagues (2012), and before them Chamberlain (2008) and Tandon and colleagues (2005), recommended that these barriers be dealt with through more formal, intensive, and ongoing skills-based training for home visitors who are dealing with IPV risk assessment, communication about risks, safety planning, and tailoring interventions; and that this training should include helping home visitors become familiar with the legal options and social supports available for mothers experiencing IPV. Chamberlin (2008) also recommended that EPHV programs should develop partnerships with other community organizations in order to help facilitate necessary referrals and interventions. Another recommendation was the design of detailed screening protocols and intervention curricula specific to the particular circumstances of EPHV that take into account the barriers to addressing IPV in a home visitation context (Chamberlain, 2008; Tandon et al., 2005). In general, EPHV programs have been observed to have a potential role in reducing the incidence of IPV following childbirth; however, there is a need for more research to determine the most effective ways of implementing this practice, and for more work to be done in optimizing these programs to best meet the specific needs of mothers at risk of IPV.

\section{Interventions for Couples}

Violence prevention counselling and education programs for expectant couples have also been noted in the IPV prevention literature, but this approach does not appear to be widely researched: we found only two articles on these topics, both published in the same year. Halford, 
International Journal of Child, Youth and Family Studies (2016) 7(3/4): 324-342

Petch, Creedy, and Gamble (2011) examined prevalence rates of IPV in couples who were expecting their first child and attending couple relationship education. They found that $32 \%$ of expectant couples reported at least one incident of violence in the past year, and 7\% reported injury resulting from IPV. Halford et al. suggested that these prevalence rates indicate that couples relationship education programs should include training in healthy communication and conflict management, with the express purpose of reducing the risk of IPV. Florsheim, McArthur, Hudak, Heavin, and Burrow-Sanchez (2011) examined the impact of the Young Parenthood Program, a co-parenting counselling program for young expectant parents, on IPV. They found that 3 months after the birth couples attending the program were significantly less likely to report occurrence of IPV than were those in the control group; however, this difference was not sustained in the 18month follow-up. Still, Florsheim et al. suggest that the 3-month results can be seen as encouraging and that counselling programs may address some of the risk factors for IPV during the transition to parenthood, although program improvements and further research are needed.

\section{Interventions for Perpetrators}

Our review located a small body of literature focusing on fathers as perpetrators of IPV that situated these men as responsible for their own violent behaviour and its consequences for their intimate partners and their children. In a marked contrast to what we found in the literature on working with female IPV victims, which provided empirical and evaluative data to help inform practice, much of the research on male perpetrators is based on qualitative inquiries that foreground male perspectives but do not examine actual intervention practices.

The dearth of programming for male perpetrators of IPV is well noted in the more recent research on this issue (Alderson, Westmarland, \& Kelly, 2013; Stanley, Graham-Kevan, \& Borthwick, 2012; Stover, 2013). Further, within that literature the absence of violence prevention approaches that focus on fatherhood is particularly striking in light of statistics indicating that $84 \%$ of men arrested for IPV have some type of fathering role; and that in $82 \%$ of cases, these relationships continued after arrest (Salisbury, Henning, \& Holdford, 2009). Also striking is that in their analysis of existing IPV perpetrator programs, Alderson et al. (2013) found that half of the programs they examined did not provide any services for children, and only three organizations worked with the children of fathers in the program. Still, we were able to locate some early research in this area and we present it below.

\section{Transition to Fatherhood}

The work of Condon, Boyce, and Corkindale (2004) was particularly useful to building our understanding of IPV in men who are about to become fathers. These researchers, who examined the social and biological effects of men's transition to parenthood, reported a statistically significant deterioration in men's mood, quality of social support, couple relationships, and sexual functioning during their partner's pregnancy, with little postnatal improvement. Condon and colleagues therefore suggest that this heightening of stress during the transition to parenthood is a 
International Journal of Child, Youth and Family Studies (2016) 7(3/4): 324-342

time when men would benefit from preparation and support for fatherhood, and that doing so has the potential of mitigating the risk for violence.

\section{Fatherhood as a Motivation for Change}

Fatherhood as a motivation for change was highlighted in a number of other qualitative inquiries of fathers who perpetrated violence against their partners. For example, Perel and Peled (2008) found that fathers expressed a strong desire for a closer and warmer relationship with their children, and that this desire played a significant role in their personal narratives. Fathers indicated concern for the well-being of their children and expressed ways that they intended to care for and protect them (Stover \& Spink, 2012). Many fathers saw their growth as a parent as part of an ongoing process of change that ran parallel to their efforts toward non-violence (Perel \& Peled, 2008; Veteläinen, Gröholm, \& Holma, 2013). The findings from these reports powerfully represent men's desires to be good fathers; however, some tensions arise from significant evidence that also indicated that men may not perceive the negative impact of their violent behaviours on their children, and therefore did not account for that violence in their assessment of their own parenting.

In many cases, men's talk of violence was in distinct isolation from their talk of fatherhood, avoiding any linking of these two aspects of themselves (Perel \& Peled, 2008; Veteläinen et al., 2013). Many fathers were prepared to admit that their children had been exposed to some violence, but few perceived this exposure as having a negative impact on the children (Stover \& Spink, 2012; Salisbury et al., 2009). However, this trend may be stronger for nonbiological fathers; in a comparison of biological fathers' and stepfathers' perceptions of the effects of their IPV on their children, biological fathers were more likely to report an awareness that their violence negatively impacted their children in a range of domains (Rothman, Mandel, \& Silverman, 2007). On a positive overall note, despite these differences, the majority in both the biological father and stepfather groups indicated that they were willing to stop their violence and seek help (Rothman et al., 2007).

In order to begin to determine whether fatherhood plays a role in the success rates of various male-focused IPV intervention programs, the interaction between fatherhood and the treatment type was examined in a study comparing a coordinated substance use and IPV treatment program with treatment for substance use alone (Smith Stover, McMahon, \& Easton, 2011). This study showed that combining substance use treatment with IPV treatment was significantly more effective than offering treatment for substance use alone in reducing both violence and alcohol use in the men who were not fathers, but generated no significant differences for men who were fathers (Smith Stover et al., 2011). The exact relationship between fatherhood and treatment outcomes, therefore, remains unclear. Smith Stover et al. (2011) concluded that these findings further underline the need to better understand how fatherhood plays a role in violence prevention and intervention and how this role is connected to substance abuse. In a further response to the lack of fatherhood-focused programming, Stanley and colleagues (2012) examined the impact of 
International Journal of Child, Youth and Family Studies (2016) 7(3/4): 324-342

existing programs for male perpetrators that did not explicitly address men's parenting in order to determine how these programs impacted fathers. Through a series of qualitative interviews, Stanley et al. (2012) determined that many fathers in the program were motivated to change their behaviours in order to regain custody of their children following an apprehension, and expressed the desire to become a "better father" and make a positive difference for their children. The authors therefore suggested that "men's needs to protect and maintain a positive image of themselves as fathers could function as motivation to act to change abusive behaviour" (Stanley et al., 2012, p. 272).

In response to this evidence regarding fathers' perceptions of the impact of IPV exposure on children, it has been recommended that intervention programming for fathers who perpetrate IPV should include among their aims increasing men's awareness about the negative effects of exposure for children, and prompting reflection on this behaviour as part of a larger commitment to being a "good father" (Alderson et al., 2013; Salisbury et al., 2009). Indeed, the small handful of fathers who were prepared to discuss the impact of their violence on children were those who had previously completed intensive IPV interventions (Perel \& Peled, 2008). This suggests that interventions that focus on educating perpetrators about the impact of their actions on their children are well placed within an ongoing process of change toward non-violence.

\section{Interventions for Fathers who Perpetrate IPV}

Relatively recently, the Fathers for Change program was developed to address the comorbidity of IPV and substance abuse and its impact on parenting outcomes (Stover, 2013). This program was developed with a focus on fathering, and sought to increase parenting competence as a motivation to change these men's interrelated patterns of violence and substance use. In order to evaluate the impacts of this program, Stover (2015) conducted a small sample evaluation of 18 fathers who were randomly assigned to the program and compared to a matched group receiving standard drug counselling. This evaluation showed that men in the Fathers for Change group were more likely to complete the treatment and reported greater satisfaction with the program; however, those who completed either group reduced their use of substances to the same degree and both groups had significant reductions in violence over time, although this trend was greater for the men in the Fathers for Change program. Stover's findings augur well for the Fathers for Change approach and suggest that more large-scale evaluations of the program should be completed in order to further establish its effectiveness in working with fathers who engage in IPV.

Also promising as an intervention that has the potential to reduce IPV in fathers is Strong Fathers, a psycho-educational, cognitive-behavioural approach for managing co-occurring IPV and child maltreatment (Penell, Rikard, \& Sanders-Rice, 2014). Using participants' self-assessments and an analysis of child protection data to examine the effects of the Strong Fathers program, Penell et al. found that men's desires to be "good fathers" are strongly connected to their motivations to change their patterns of violence; following completion of the Strong Fathers 
International Journal of Child, Youth and Family Studies (2016) 7(3/4): 324-342

program, the number of child protection reports involving the program participants decreased from 32 of the 53, to only 4 of the 53. It is noteworthy that three of the fathers involved in the four child protection incident reports did not complete the program, thus suggesting that full completion of the program may have reduced this number of child protection incidents even further. These results are promising and, as with those generated by the Fathers For Change study (Stover, 2015), warrant further research.

\section{Summary and Directions for Future Research}

The research that has been presented here represents the majority of the recent peerreviewed literature regarding the prevention of IPV during childbearing years, particularly with respect to preventing children's exposure to violence. This research has by and large focused on contemporary practices directed at influencing the behaviour of mothers as potential victims of IPV. Although there have been some promising findings, there remains a need for more largescale, high-quality trials in order to determine the most effective methods of screening and intervention with mothers. There is also a need for the development of more IPV-specific training of professionals and a protocol for assessing risk in order to improve the rates of screening and the quality of responses to IPV disclosure. Across the board, the literature suggests that all professionals interacting with pregnant women and recent mothers need to become familiar with available community resources in order to develop a meaningful response to IPV and to help women access appropriate supports.

We also note that the research on the prevention of female IPV victimization was largely characterized by contexts of women's medical care and home visitation programs focused on mothers. We believe that this may be largely a result of the immediate access to mothers and children during these medical visits. Although we acknowledge the practicality of this approach, and the intention of creating safe spaces for battered women and their children, we question the extent to which this contributes to discourses that focus on the victimhood of women and represent women's choices as a determining factor in children's exposure to violence. As Featherstone and Peckover (2007) point out, strategies directed at influencing the behaviour of IPV victims position these mothers as responsible for managing and mitigating risk to themselves and their children; and, in shifting attention away from men's behaviours, miss a significant opportunity to engage with men as potential perpetrators of IPV, specifically as they transition into the role of father. More services for fathers would not only constitute a more comprehensive response to IPV, but would more effectively address the safety needs of women and children by addressing the problem at its immediate source. In a call for more perpetrator programming that specifically addresses men as fathers, Alderson et al. (2013) contend:

If perpetrator programmes are successful in changing [violent] men's ways of engaging with their children, and [these] men can safely be involved in their children's lives, then they are integral to an overall social work response to the safeguarding and protecting of children who live with domestic violence. (p. 191) 
International Journal of Child, Youth and Family Studies (2016) 7(3/4): 324-342

Based on the results of our search, we believe that research on programs for IPV prevention for fathers is still in its infancy. Fortunately, we were able to locate some promising findings indicating that fatherhood is a key motivator in men's processes of change toward cessation of violence (Penell et al., 2014; Stover, 2015). Unfortunately, we were not able to uncover work focused on men that explored the development and evaluation of strategies to prevent their engagement in IPV during their transition to fatherhood. We posit that, for men, the time during which they and their partners are expecting a child is as opportune a moment to work on IPV prevention as it is for women, given that this has been identified as a period of stress and uncertainty, and such programs would help prepare them for the challenges of fatherhood (Condon et al., 2004). This is an area of IPV prevention that has been neglected, but presents a rich opportunity to provide a response that avoids placing the responsibility for mitigation of violence on mothers, and has the potential to increase the safety and well-being of all members of the family. 
International Journal of Child, Youth and Family Studies (2016) 7(3/4): 324-342

\section{References}

Alderson, S., Westmarland, N., \& Kelly, L. (2013). The need for accountability to, and support for, children of men on domestic violence perpetrator programmes. Child Abuse Review, 22(3), 182-193.

Anderson, B. A., Marshak, H. H., and Hebbeler, D. L. (2002), Identifying intimate partner violence at entry to prenatal care: Clustering routine clinical information. Journal of Midwifery \& Women's Health, 47, 353-359.

Andresen, M. A., \& Linning, S. J. (2014). Beginning to understand the economic costs of children's exposure to intimate partner violence. International Journal of Child, Youth and Family Studies, 5(4), 588-608.

Artz, S., Jackson, M. A., Rossiter, K. R., Nijdam-Jones, A., Géczy, I., \& Porteous, S. (2014). A comprehensive review of the literature on the impact of exposure to intimate partner violence for children and youth. International Journal of Child, Youth and Family Studies, 5(4), 493587.

Bacchus, L., Mezey, G., Bewley, S., \& Haworth, A. (2004). Prevalence of domestic violence when midwives routinely enquire in pregnancy. BJOG: An International Journal of Obstetrics \& Gynaecology, 111(5), 441-445.

Baird, K., Salmon, D., \& White, P. (2013). A five year follow-up study of the Bristol pregnancy domestic violence programme to promote routine enquiry. Midwifery, 29(8), 1003-1010.

Bair-Merritt, M. H., Jennings, J. M., Chen, R., Burrell, L., McFarlane, E., Fuddy, L., \& Duggan, A. K. (2010). Reducing maternal intimate partner violence after the birth of a child: A randomized controlled trial of the Hawaii Healthy Start Home Visitation Program. Archives of Pediatrics \& Adolescent Medicine, 164(1), 16-23.

Bianchi, A. L., McFarlane, J., Nava, A., Gilroy, H., Maddoux, J., \& Cesario, S. (2014). Rapid assessment to identify and quantify the risk of intimate partner violence during pregnancy. Birth, 41(1), 88-92.

Borowsky, I. W., \& Ireland, M. (2002). Parental screening for intimate partner violence by pediatricians and family physicians. Pediatrics, 110(3), 509-516.

Canadian Centre for Justice Statistics. (2015). Family violence in Canada: A statistical profile, 2013 (Catalogue no. 85-002-X ISSN 1209-6393). Juristat. Ottawa, ON: Statistics Canada.

Chamberlain, L. (2008). Ten lessons learned in Alaska: Home visitation and intimate partner violence. Journal of Emotional Abuse, 8(1-2), 205-216. 
International Journal of Child, Youth and Family Studies (2016) 7(3/4): 324-342

Condon, J. T., Boyce, P., \& Corkindale, C. J. (2004). The first-time fathers study: A prospective study of the mental health and wellbeing of men during the transition to parenthood. Australian and New Zealand Journal of Psychiatry, 38(1-2), 56-64.

Cripe, S. M., Sanchez, S. E., Sanchez, E., Quintanilla, B. A., Alarcon, C. H., Gelaye, B., \& Williams, M. A. (2010). Intimate partner violence during pregnancy: A pilot intervention program in Lima, Peru. Journal of Interpersonal Violence, 25(11), 2054-2076.

Eddy, T., Kilburn, E., Chang, C., Bullock, L., \& Sharps, P. (2008). Facilitators and barriers for implementing home visit interventions to address intimate partner violence: Town and gown partnerships. Nursing Clinics of North America, 43(3), 419-435.

Erickson, M. J., Hill, T. D., \& Siegel, R. M. (2001). Barriers to domestic violence screening in the pediatric setting. Pediatrics, 108(1), 98-102.

Featherstone, B., \& Peckover, S. (2007). Letting them get away with it: Fathers, domestic violence and child welfare. Critical Social Policy, 27(2), 181-202.

Florsheim, P., McArthur, L., Hudak, C., Heavin, S., \& Burrow-Sanchez, J. (2011). The Young Parenthood Program: Preventing intimate partner violence between adolescent mothers and young fathers. Journal of Couple \& Relationship Therapy, 10(2), 117-134.

Halford, W. K., Petch, J., Creedy, D. K., \& Gamble, J. (2011). Intimate partner violence in couples seeking relationship education for the transition to parenthood. Journal of Couple \& Relationship Therapy, 10(2), 152-168.

Humphreys, J., Tsoh, J. Y., Kohn, M. A., \& Gerbert, B. (2011). Increasing discussions of intimate partner violence in prenatal care using Video Doctor plus Provider Cueing: A randomized, controlled trial. Women's Health Issues, 21(2), 136-144.

Jack, S. M., Ford-Gilboe, M., Wathen, C. N., Davidov, D. M., McNaughton, D. B., Coben, J. H., ... \& NFP IPV Research Team. (2012). Development of a nurse home visitation intervention for intimate partner violence. BMC Health Services Research, 12(1), 50.

Jackson, M.A., \& Artz, S. (Eds.). (2014). In harm's way [Special issue on the impacts and costs of witnessing intimate partner violence]. International Journal of Child, Youth and Family Studies, 5(4), 493-608.

Kataoka, Y., Yaju, Y., Eto, H., \& Horiuchi, S. (2010). Self-administered questionnaire versus interview as a screening method for intimate partner violence in the prenatal setting in Japan: A randomised controlled trial. BMC Pregnancy and Childbirth, 10(1), 84. 
International Journal of Child, Youth and Family Studies (2016) 7(3/4): 324-342

Kiely, M., El-Mohandes, A. A., El-Khorazaty, M. N., \& Gantz, M. G. (2010). An integrated intervention to reduce intimate partner violence in pregnancy: A randomized trial. Obstetrics and Gynecology, 115(2 Pt. 1), 273-283.

Kramer, A., Nosbusch, J. M., \& Rice, J. (2012). Safe Mom, Safe Baby: A collaborative model of care for pregnant women experiencing intimate partner violence. The Journal of Perinatal \& Neonatal Nursing, 26(4), 307-316.

Lazenbatt, A., Taylor, J., \& Cree, L. (2009). A healthy settings framework: An evaluation and comparison of midwives' responses to addressing domestic violence. Midwifery, 25(6), 622636.

Marchant, S., Davidson, L. L., Garcia, J., \& Parsons, J. E. (2001). Addressing domestic violence through maternity services: Policy and practice. Midwifery,17(3), 164-170.

McFarlane, J., Soeken, K., \& Wiist, W. (2000). An evaluation of interventions to decrease intimate partner violence to pregnant women. Public Health Nursing, 17(6), 443-451.

Mejdoubi, J., van den Heijkant, S. M., van Leerdam, F. M., Heymans, M. W., Hirasing, R. A., \& Crijnen, A. M. (2013). Effect of nurse home visits vs. usual care on reducing intimate partner violence in young high-risk pregnant women: A randomized controlled trial. PLOS ONE, $8(10), 1$.

Olds, D., Robinson, J., Pettitt, L., Luckey, D., Holmberg, J., Ng, R., ... \& Henderson, C. J. (2004). Effects of home visits by paraprofessionals and by nurses: Age 4 follow-up results of a randomized trial. Pediatrics, 114(6), 1560-1568.

Pennell, J., Rikard, R. V., \& Sanders-Rice, T. (2014). Family violence: Fathers assessing and managing their risk to children and women. Children and Youth Services Review, 47, 36-45.

Perel, G., \& Peled, E. (2008). The fathering of violent men: Constriction and yearning. Violence Against Women, 14(4), 457-482.

Rådestad, I., Rubertsson, C., Ebeling, M., \& Hildingsson, I. (2004). What factors in early pregnancy indicate that the mother will be hit by her partner during the year after childbirth? A nationwide Swedish survey. Birth: Issues In Perinatal Care, 31(2), 84-92.

Reichenheim, M. E., Patricio, T. F., \& Moraes, C. L. (2008). Detecting intimate partner violence during pregnancy: Awareness-raising indicators for use by primary healthcare professionals. Public Health, 122(7), 716-724.

Rothman, E. F., Mandel, D. G., \& Silverman, J. G. (2007). Abusers’ perceptions of the effect of their intimate partner violence on children. Violence Against Women, 13(11), 1179-1191. 
International Journal of Child, Youth and Family Studies (2016) 7(3/4): 324-342

Salisbury, E. J., Henning, K., \& Holdford, R. (2009). Fathering by partner-abusive men: Attitudes on children's exposure to interparental conflict and risk factors for child abuse. Child Maltreatment, 14(3), 232-242.

Salmon, D., Murphy, S., Baird, K., \& Price, S. (2006). An evaluation of the effectiveness of an educational programme promoting the introduction of routine antenatal enquiry for domestic violence. Midwifery, 22(1), 6-14.

Sharps, P., Alhusen, J. L., Bullock, L., Bhandari, S., Ghazarian, S., Udo, I. E., \& Campbell, J. (2013). Engaging and retaining abused women in perinatal home visitation programs. Pediatrics, 132(Suppl. 2), S134-S139.

Sinha, M. (2012). Family violence in Canada: A statistical profile, 2010 (Catalogue no. 85-002-X). Juristat. Ottawa, ON: Statistics Canada.

Sinha, M. (2013). Section 3: Intimate partner violence (Catalogue no. 85-002-X). Juristat - Family Violence in Canada: A Statistical Profile, 2011. Ottawa, ON: Statistics Canada.

Smith Stover, C., McMahon, T. J., \& Easton, C. (2011). The impact of fatherhood on treatment response for men with co-occurring alcohol dependence and intimate partner violence. The American Journal Of Drug And Alcohol Abuse, 37(1), 74-78.

Stanley, N., Graham-Kevan, N., \& Borthwick, R. (2012). Fathers and domestic violence: Building motivation for change through perpetrator programmes. Child Abuse Review, 21(4), 264-274.

Stover, C. S. (2013). Fathers for change: A new approach to working with fathers who perpetrate intimate partner violence. Journal of the American Academy of Psychiatry and the Law Online, 41(1), 65-71.

Stover, C. S. (2015). Fathers for change for substance use and intimate partner violence: Initial community pilot. Family Process, 54(4), 600-609.

Stover, C. S., \& Spink, A. (2012). Affective awareness in parenting of fathers with co-occurring substance abuse and intimate partner violence. Advances in Dual Diagnosis, 5(2), 74-85.

Taft, A. J., Small, R., Hegarty, K. L., Watson, L. F., Gold, L., \& Lumley, J. A. (2011). Mothers’ Advocates In the Community (MOSAIC) - non-professional mentor support to reduce intimate partner violence and depression in mothers: A cluster randomised trial in primary care. BMC Public Health, 11(1), 178.

Tandon, S. D., Parillo, K. M., Jenkins, C., \& Duggan, A. K. (2005). Formative evaluation of home visitors' role in addressing poor mental health, domestic violence, and substance abuse among low-income pregnant and parenting women. Maternal and Child Health Journal, 9(3), 273-283. 
International Journal of Child, Youth and Family Studies (2016) 7(3/4): 324-342

Thackeray, J. D., Hibbard, R., \& Dowd, M. D. (2010). Intimate partner violence: The role of the pediatrician. Pediatrics, 125(5), 1094-1100.

Tiwari, A., Leung, W. C., Leung, T. W., Humphreys, J., Parker, B., \& Ho, P. C. (2005). A randomised controlled trial of empowerment training for Chinese abused pregnant women in Hong Kong. BJOG: An International Journal of Obstetrics \& Gynaecology, 112(9), 12491256.

Veteläinen, A., Grönholm, H., \& Holma, J. (2013). Discussions of fatherhood in male batterer treatment group. SAGE Open, 3(2), 1-10. 